\title{
A $\beta$ INVARIANT FOR GREEDOIDS AND ANTIMATROIDS
}

\author{
GARY GORDON \\ Department of Mathematics \\ Lafayette College \\ Easton, PA 18042 \\ gordong@lafayette.edu \\ Submitted: November 12, 1996; Accepted: March 28, 1997.
}

\begin{abstract}
We extend Crapo's $\beta$ invariant from matroids to greedoids, concentrating especially on antimatroids. Several familiar expansions for $\beta(G)$ have greedoid analogs. We give combinatorial interpretations for $\beta(G)$ for simplicial shelling antimatroids associated with chordal graphs. When $G$ is this antimatroid and $b(G)$ is the number of blocks of the chordal graph $G$, we prove $\beta(G)=1-b(G)$.
\end{abstract}

\section{INTRODUCTION}

In this paper, we define a $\beta$ invariant for antimatroids and greedoids. This continues the program of extending matroid invariants to greedoids which was begun in [17], where the 2-variable Tutte polynomial was defined for greedoids. The Tutte polynomial has been studied for several important classes of greedoids, including partially ordered sets, rooted graphs, rooted digraphs and trees. Recently, the onevariable characteristic polynomial [19]] was extended from matroids to greedoids.

Crapo's $\beta$ invariant for matroids was introduced in [12] If $M$ is a matroid, then $\beta(M)$ is a non-negative integer which gives information about whether $M$ is connected and whether $M$ is the matroid of a series-parallel network. In particular, $\beta(M)=0$ iff $M$ is disconnected (or $M$ consists of a single loop) [12] and $\beta(M)=1$ iff $M$ is the matroid of a series-parallel network (or $M$ consists of a single isthmus) [6]. More recently (see 24]), interest has focused on characterizing matroids with small $\beta$. A standard reference for many of the basic properties of $\beta(M)$ is [28].

In Section 2 we give several elementary results, each of which extends a corresponding matroid result. We define $\beta(G)$ in terms of the Tutte polynomial, then show $\beta(G)$ has the same subset expansion as in the matroid case Proposition 2.1 and obeys a slightly different deletion-contraction recursion Proposition 2.2. It is still true that $\beta\left(G_{1} \oplus G_{2}\right)=0$, but the converse is false Proposition 2.3 and Example 2.1).

Section 3 applies the activities approach of [18] to $\beta(G)$. This approach allows us to connect $\beta(G)$ to the poset $\left(\mathcal{F}_{\emptyset}, \subseteq\right)$, where $\mathcal{F}_{\emptyset}$ is the collection of feasible sets having no external activity. This is the greedoid version of the broken circuit complex of a matroid, a well studied object on its own [4,7,8]. We get a Whitney number expansion for $\beta(G)$ (as in the matroid case) and also give a matching result

1991 Mathematics Subject Classification. Primary: 05B.

Key words and phrases. Greedoid, antimatroid, $\beta$ invariant. 
2 The electronic Journal of Combinatorics 4 (1997), \#R13

for $\left(\mathcal{F}_{\emptyset}, \subseteq\right)$. As a consequence of this result, we derive several expansions for $\beta(G)$ in terms of the family $\mathcal{F}_{\emptyset}$.

In Section 4, we concentrate on antimatroids. Antimatroids have been studied by a number of people in connection with convexity, algorithm design and greedoids. In fact, antimatroids have been rediscovered several times, having been introduced by Dilworth in the 1940's. See [22] and [5, pages 343-4] for short and interesting accounts of the development of antimatroids.

For antimatroids, the poset $\left(\mathcal{F}_{\emptyset}, \subseteq\right)$ is a join-distributive join semilattice. We use this additional structure to derive a Möbius function formulation and several convex set expansions for $\beta(G)$.

Section 5 is devoted to an interesting example, simplicial shelling in chordal graphs. The main theorem of this section, Theorem 5.1 shows that if $G$ is the antimatroid associated with a chordal graph and $b(G)$ is the number of blocks of $G$, then $\beta(G)=1-b(G)$.

We take the view that the definition of $\beta(G)$ considered here is probably the most reasonable generalization from matroids to greedoids. The fact that so many matroidal properties of $\beta$ have greedoid analogs is strong evidence for this position. In addition, there are several interesting combinatorial interpretations (not explored here) for $\beta(G)$ when $G$ is a rooted graph, digraph, tree, poset or convex point set. Some of these interpretations are closely related to matroidal or graphical properties of $\beta$. This lends support to our view that the Tutte and characteristic polynomials studied in $[11,14,15,16,17,18,19]$ are (in some sense) also the 'right' generalizations to greedoids.

\section{DEFINITIONS AND FUNDAMENTAL PROPERTIES}

We assume the reader is familiar with matroid theory. Define a greedoid as follows:

Definition 2.1. A greedoid $G$ on the ground set $E$ is a pair $(E, \mathcal{F})$ where $|E|=n$ and $\mathcal{F}$ is a family of subsets of $E$ satisfying

1. For every non-empty $X \in \mathcal{F}$ there is an element $x \in X$ such that $X-\{x\} \in \mathcal{F}$;

2. For $X, Y \in \mathcal{F}$ with $|X|<|Y|$, there is an element $y \in Y-X$ such that $X \cup\{y\} \in \mathcal{F}$.

A set $F \in \mathcal{F}$ is called feasible. The family of independent sets in a matroid satisfy these requirements, so every matroid is a greedoid. One significant difference between matroids and greedoids is that every subset of an independent set is independent in a matroid, but a feasible set in a greedoid will have non-feasible subsets in general. As with matroids, the rank of a set $A$, denoted $r(A)$, is the size of the largest feasible subset of $A$ :

$$
r(A)=\max _{S \in \mathcal{F}}\{|S|: S \subseteq A\} .
$$

An extensive introduction to greedoids can be found in [5] or [23]

We now define two polynomial invariants for greedoids.

Definition 2.2. Let $G$ be a greedoid on the ground set $E$.

1. Tutte polynomial:

$$
f(G ; t, z)=\sum_{S \subseteq E} t^{r(G)-r(S)} z^{|S|-r(S)} .
$$


2. Characteristic polynomial:

$$
p(G ; \lambda)=(-1)^{r(G)} f(G ;-\lambda,-1) .
$$

The Tutte polynomial for greedoids was introduced in [17] and has been studied for various greedoid classes. A deletion-contraction recursion (Theorem 3.2 of [17]) holds for this Tutte polynomial, as well as an activities interpretation (Theorem 3.1 of [18]]. The characteristic polynomial was studied in [19].

We now define $\beta(G)$ for a greedoid $G$.

Definition 2.3. Let $G$ be a greedoid on the ground set $E$ with Tutte polynomial $f(G ; t, z)$. Then

$$
\beta(G)=\frac{\partial f}{\partial t}(-1,-1)
$$

We could equally well define $\beta(G)$ in terms of $p(G ; \lambda): \beta(G)=(-1)^{r(G)-1} p^{\prime}(1)$.

The following proposition follows directly from our definition and has precisely the same form for matroids.

Proposition 2.1 (Subset sum). Let $G$ be a greedoid. Then

$$
\beta(G)=(-1)^{r(G)} \sum_{S \subseteq E}(-1)^{|S|} r(S) .
$$

The next proposition follows from applying $\frac{\partial}{\partial t}$ to the deletion-contraction recursion in Proposition 3.2 of [17]

Proposition 2.2 (Deletion-contraction). Let $G$ be a greedoid and let $\{e\} \in \mathcal{F}$. Then

$$
\beta(G)=\beta(G / e)+(-1)^{r(G)-r(G-e)} \beta(G-e) .
$$

We remark that since $r(G-e)=r(G)$ for all non-isthmuses $e$ in a matroid $G$, the formula above reduces to the familiar $\beta(G)=\beta(G-e)+\beta(G / e)$ for matroids. We also note that, unlike the matroid case, $\beta(G)<0$ is possible (as the coefficient $(-1)^{r(G)-r(G-e)}$ may be negative). See Section 5 .

Proposition 2.3 (Direct sum property). $\beta\left(G_{1} \oplus G_{2}\right)=0$.

Proof. Just apply $\frac{\partial}{\partial t}$ to the equation $f\left(G_{1} \oplus G_{2} ; t, z\right)=f\left(G_{1} ; t, z\right) f\left(G_{2} ; t, z\right)$ (Proposition 3.7 of $[17]$ and note that $f(G ;-1,-1)=0$ for any non-empty greedoid $G$.

Recall that an element $e$ is a greedoid loop if $e$ is in no feasible set.

Corollary 2.4. If $G$ contains greedoid loops, then $\beta(G)=0$.

Proposition 2.3 is half of Crapo's important connectivity result for matroids (Theorem 7.3.2 of [28]): $\beta(M)=0$ if and only if $M=M_{1} \oplus M_{2}$ (and $M$ is not a loop). The converse of Proposition 2.3 is false for greedoids: It is possible for $\beta(G)=0$ when $G$ does not decompose as a direct sum of smaller greedoids. This is the point of the next example.

Example 2.1. Let $G=(E, \mathcal{F})$ be a greedoid with $E=\{a, b, c\}$ and feasible sets $\mathcal{F}=\{\emptyset,\{a\},\{b\},\{a, c\},\{b, c\},\{a, b, c\}\}$. Then the reader can check that $f(G ; t, z)=$ $(t+1)\left(t^{2}(z+1)+t+1\right)$, so $\beta(G)=0$ from Definition 2.3. But it is easy to show that $G$ is not a direct sum of two smaller greedoids. 
4 The electronic Journal of Combinatorics 4 (1997), \#R13

\section{Activities EXPANSIONS}

Basis activities formed the foundation for Tuttes original work on the twovariable dichromatic graph polynomial which now bears his name [27]. In [18], a notion of external activity for feasible sets in a greedoid is developed. We now briefly recall the definitions and fundamental results we will need.

A computation tree $T_{G}$ for a greedoid $G$ is a recursively defined, rooted, binary tree in which each vertex of $T_{G}$ is labeled by a minor of $G$. More precisely, if the vertex $v$ in $T_{G}$ receives label $H$ for some minor $H$ of $G$, we label the two children of $v$ by $H-e$ and $H / e$, where $\{e\}$ is some feasible set in $H$. The process terminates when $H$ consists solely of greedoid loops. We label the root of $T_{G}$ with $G$ and note that $T_{G}$ obviously depends on the order in which elements are deleted and contracted.

When $T_{G}$ is a computation tree for a greedoid $G$, there is a natural bijection between the feasible sets of $G$ and the terminal vertices of $T_{G}$ which is given by listing the elements of $G$ which are contracted in arriving at the specified terminal vertex. Define the external activity of a feasible set $F$ with respect to the tree $T_{G}$ by $\operatorname{ext}_{T}(F)=A$ where $A \subseteq E$ is the collection of greedoid loops which labels the terminal vertex corresponding to $F$. Thus, $\operatorname{ext}_{T}(F)$ consists of the elements of $G$ which were neither deleted nor contracted along the path from the root of $T_{G}$ to the terminal vertex corresponding to $F$.

Proposition 3.1 (Feasible set expansion). Let $T_{G}$ be a computation tree for $G$ and let $\mathcal{F}_{\emptyset}$ denote the set of all feasible sets of $G$ having no external activity. Then

$$
\beta(G)=(-1)^{r(G)} \sum_{F \in \mathcal{F}_{\emptyset}}(-1)^{|F|-1}(r(G)-|F|) .
$$

Proof. This follows from Theorem 3.1 of [18] and our definition.

Since $r(G)-|F|=0$ precisely when $F$ is a basis for $G$, we could restrict our sum in Proposition 3.1 to all non-bases in $\mathcal{F}_{\emptyset}$.

We are interested in the structure of the ranked poset $\left(\mathcal{F}_{\emptyset}, \subseteq\right)$. When $M$ is a matroid, the family $\mathcal{F}_{\emptyset}$ forms a simplicial complex, called the broken circuit complex of $M$. Although this structure does not generalize to greedoids, we can still interpret some of the matroidal properties of the broken circuit complex in the more general context of greedoids.

For matroids, the Whitney numbers of the first kind are the face enumerators for the broken circuit complex [3]. In [19], we define Whitney numbers of the first kind for a greedoid $G$ via the characteristic polynomial (see Definition 2.2(2)).

Definition 3.1. If $p(G ; \lambda)=\sum_{k=0}^{r(G)} w_{k} \lambda^{r(G)-k}$, then the coefficient $w_{k}$ is the $k^{t h}$ Whitney number of the first kind for $G$.

In [19], we show that if $T_{G}$ is a computation tree for a greedoid $G$, then $(-1)^{k} w_{k}$ equals the number of feasible sets in $\mathcal{F}_{\emptyset}$ of cardinality $k$, exactly as in the matroid case. Thus the number of such feasible sets does not depend on $T_{G}$. For matroids, the sequence $\left\{(-1)^{k} w_{k}\right\}$ (sometimes written $\left\{w_{k}^{+}\right\}$) is one of many sequences associated with matroids which is conjectured to be unimodal. (See [2] for an account of some results concerning this and other related conjectures.) This is false for greedoids, however - the sequence of Whitney numbers given in Example 2 of [19] is not unimodal. 
The next proposition generalizes another matroid expansion of $\beta(G)$. The proof follows immediately from the definitions.

Proposition 3.2 (Whitney numbers expansion). Let $w_{k}$ be the $k^{\text {th }}$ Whitney number of $G$. Then

$$
\beta(G)=\sum_{k>0}(-1)^{k-1} k w_{k}
$$

We now give a structural result for $\left(\mathcal{F}_{\emptyset}, \subseteq\right)$.

Theorem 3.1. Let $T_{G}$ be a computation tree for a greedoid $G$ and let $\left(\mathcal{F}_{\emptyset}, \subseteq\right)$ be the ranked poset of feasible sets with no external activity. Then the Hasse diagram for $\left(\mathcal{F}_{\emptyset}, \subseteq\right)$ has a perfect matching (in the graph theoretic sense).

Proof. Let $F \in \mathcal{F}_{\emptyset}$. Then $\operatorname{ext}_{T}(F)=\emptyset$, so the terminal vertex $v_{F}$ of $T_{G}$ which corresponds to $F$ is an empty greedoid. Consider the vertex $w$ in $T_{G}$ which is the parent of $v_{F}$. Let $H$ be the greedoid minor which corresponds to $w$. Then $|H|=1$, since either $H-e$ or $H / e$ is empty. Further, $r(H)=1$ since otherwise $w$ would be a terminal vertex of $T_{G}$. Thus $H=\{e\}$ and $r(e)=1$.

There are two possibilities for which child of $H$ the vertex $v_{F}$ can be. If $v_{F}$ corresponds to $H-e$, then let $u_{F}$ correspond to $H / e$ in $T_{G}$. If $v_{F}$ corresponds to $H / e$, then let $u_{F}$ correspond to $H-e$ in $T_{G}$. In the former case, the feasible set corresponding to $u_{F}$ covers $v_{F}$ in the poset $\left(\mathcal{F}_{\emptyset}, \subseteq\right)$; in the latter case, the covering relation is reversed. In either case, these two feasible sets are joined by an edge in the Hasse diagram of $\left(\mathcal{F}_{\emptyset}, \subseteq\right)$. This pairing of the feasible sets in $\mathcal{F}_{\emptyset}$ gives us the desired matching.

Corollary 3.3. $\left|\mathcal{F}_{\emptyset}\right|$ is even for any computation tree $T_{G}$.

By Theorem 3.1 of [18], $f(G ; 1,-1)=\left|\mathcal{F}_{\emptyset}\right|$ for the Tutte polynomial $f(G ; t, z)$. Thus we also obtain $f(G ; 1,-1)$ is even for any greedoid $G$. This is easy to prove in other ways. It is interesting to note that when $G$ is a graph, a celebrated result of Stanley [25] shows the evaluation $f(G ; 1,-1)$ equals the number of acyclic orientations of $G$, which is obviously even. (The matroid associated to $G$ here is the usual cycle matroid.)

We now use the matching in Theorem 3.1 to obtain another expression for $\beta(G)$.

Proposition 3.4. Let $T_{G}$ be a computation tree for a greedoid $G$ and let $\mathcal{F}_{\text {min }} \subseteq \mathcal{F}_{\emptyset}$ denote the set of all feasible sets which are the minimal elements of the matching given in Theorem 3.1. Then

$$
\beta(G)=(-1)^{r(G)} \sum_{F \in \mathcal{F}_{\text {min }}}(-1)^{|F|-1} .
$$

Proof. Let $F \in \mathcal{F}_{\min }$. Then by the proof of Theorem 3.1, there is an element $e_{F}$ such that $F \cup\left\{e_{F}\right\} \in \mathcal{F}_{\text {min }}$. Then by Proposition 3.1

$$
\begin{aligned}
\beta(G) & =(-1)^{r(G)} \sum_{F \in \mathcal{F}_{\emptyset}}(-1)^{|F|-1}(r(G)-|F|) \\
& =(-1)^{r(G)} \sum_{F \in \mathcal{F}_{\text {min }}}\left[(-1)^{|F|-1}(r(G)-|F|)+(-1)^{\left|F \cup\left\{e_{F}\right\}\right|-1}\left(r(G)-\left|F \cup\left\{e_{F}\right\}\right|\right)\right] \\
& =(-1)^{r(G)} \sum_{F \in \mathcal{F}_{\text {min }}}(-1)^{|F|-1} .
\end{aligned}
$$


6 The electronic journal of Combinatorics 4 (1997), \#R13

When $M$ is a matroid, a fixed order can always be used throughout the construction of the computation tree $T_{M}$. Let $e(\neq$ loop) be the last element encountered in a given (fixed) ordering of the elements of $M$. (This corresponds to $e$ being the first element in the order in the usual treatment of matroid activities, where we operate on the elements of $M$ in reverse order.) The family of all subsets of $E-e$ that contain no broken circuits is called the reduced broken circuit complex. Then $\left(\mathcal{F}_{\text {min }}, \subseteq\right)$ in Proposition 3.4 is the reduced broken circuit complex of $M$ and the matching given in the proof of Proposition 3.4 shows that the broken circuit complex $\left(\mathcal{F}_{\emptyset}, \subseteq\right)$ is a topological cone over the reduced complex $\left(\mathcal{F}_{\text {min }}, \subseteq\right)$ with apex $e$. (See Theorem 7.4 .2 (iii) of [3].)

The next two expansions for $\beta(G)$ are consequences of Proposition 3.4.

Corollary 3.5. Let $T_{G}$ be a computation tree for a greedoid $G$ and let $\mathcal{F}_{\text {max }} \subseteq \mathcal{F}_{\emptyset}$ denote the set of all feasible sets which are the maximal elements of the matching given in Theorem 3.1. Then

$$
\beta(G)=(-1)^{r(G)} \sum_{F \in \mathcal{F}_{\max }}(-1)^{|F|} .
$$

Corollary 3.6. Let $T_{G}$ be a computation tree for a greedoid $G$ and let $\mathcal{M}$ be any perfect matching in the Hasse diagram of $\left(\mathcal{F}_{\emptyset}, \subseteq\right)$. Let $M_{1} \subseteq \mathcal{F}_{\emptyset}$ denote the set of all feasible sets which are the minimal elements of the matching $\mathcal{M}$ and let $M_{2} \subseteq \mathcal{F}_{\emptyset}$ denote the set of all feasible sets which are the maximal elements of $\mathcal{M}$. Then

1. $\beta(G)=(-1)^{r(G)} \sum_{F \in M_{1}}(-1)^{|F|-1}$,

2. $\beta(G)=(-1)^{r(G)} \sum_{F \in M_{2}}(-1)^{|F|}$.

Proof. (1) Recall that the poset $\left(\mathcal{F}_{\emptyset}, \subseteq\right)$ is ranked. Let $\mathcal{F}_{\text {min }}$ be defined as in the proof of Proposition 3.4 and let $M_{1}(k)$ and $\mathcal{F}_{\min }(k)$ denote the number of feasible sets of rank $k$ in the families $M_{1}$ and $\mathcal{F}_{\text {min }}$, resp. We will show $M_{1}(k)=\mathcal{F}_{\text {min }}(k)$ for all $k$ by induction.

To simplify notation, let $a_{k}$ be the number of feasible sets in $\mathcal{F}_{\emptyset}$ of size $k$ (so $a_{k}=w_{r-k}^{+}$, where $w_{i}^{+}$is the (unsigned) $i^{t h}$ Whitney number for $G$ and $r=r(G)$ ). Let $s=\min \left\{k: a_{k}>0\right\}$. Then $M_{1}(k)=\mathcal{F}_{\text {min }}(k)=0$ for $k<s$. We begin the induction for $k=s$. But $\mathcal{M}$ is a perfect matching, so every feasible set of size $s$ must be represented in $\mathcal{M}$ as a minimal member (since $a_{k}=0$ for $k<s$ ). Thus $M_{1}(s)=\mathcal{F}_{\min }(s)$.

Now assume $k>s$. Then $\mathcal{M}$ perfect implies every feasible set of size $k$ in $\mathcal{F}_{\emptyset}$ is either minimal in the matching (and so contributes to $M_{1}(k)$ ) or maximal in the matching (so it contributes to $M_{1}(k-1)$ ). Thus $M_{1}(k)=a_{k}-M_{1}(k-1)=$ $a_{k}-\mathcal{F}_{\text {min }}(k-1)=\mathcal{F}_{\text {min }}(k)$ by induction. This completes the proof.

(2) This is similar to (1).

We conclude this section with an example.

Example 3.1. Let $T$ be the tree appearing in Figure 1. Then the edge pruning greedoid $G=(E, \mathcal{F})$ is a greedoid on the edge set $E$ where $F \in \mathcal{F}$ if the edges of $F$ form the complement of a subtree in $T$. Then $\mathcal{F}_{\emptyset}$ is also shown in Figure 1. (Since the greedoid is an antimatroid, $\mathcal{F}_{\emptyset}$ is independent of the computation tree $T_{G}$. See the discussion in Section 4 below.) We have outlined a perfect matching in heavy lines. Thus, by Corollary 3.6(2), $\beta(T)=(-1)^{4}\left[(-1)^{2}+3(-1)^{3}+(-1)^{4}\right]=-1$. 


\section{Antimatroids}

Definition 4.1. An antimatroid $A=(E, \mathcal{F})$ is a greedoid which satisfies $F_{1}, F_{2} \in$ $\mathcal{F}$ implies $F_{1} \cup F_{2} \in \mathcal{F}$.

For an antimatroid $A$, the poset $(\mathcal{F}, \subseteq)$ of feasible sets forms a semimodular lattice. (In fact, a greedoid $G$ is an antimatroid $\operatorname{iff}(\mathcal{F}, \subset)$ is a semimodular lattice). Antimatroids are dual to convex geometries. See [5, 23] for a detailed account. For an antimatroid $A=(E, \mathcal{F})$, let $(\mathcal{C}, \subseteq)$ be the collection of convex sets in $A$, i.e., $\mathcal{C}=\{C \subseteq E: E-C \in \mathcal{F}\}$. A convex set $K \subseteq E$ is free if every subset of $K$ is also convex. The collection of all free sets, denoted $\mathcal{C}_{F}$, forms an order ideal in $(\mathcal{C}, \subseteq)$.

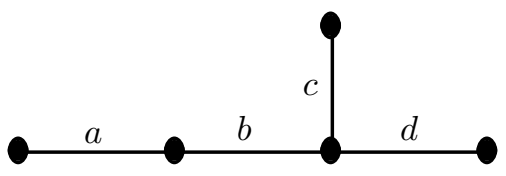

$T$

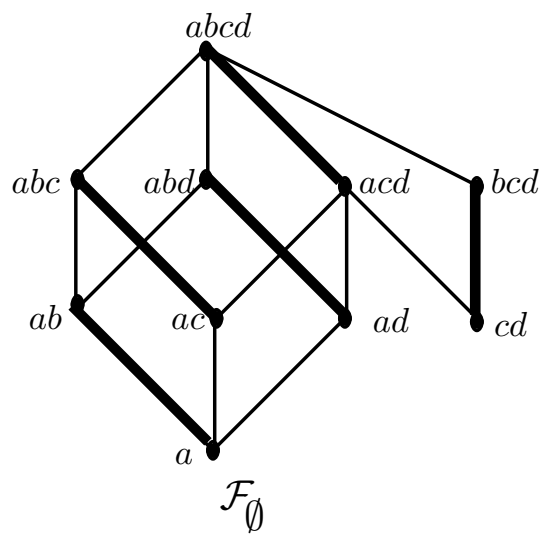

FiguRE 1.

For antimatroids, $F \cup \operatorname{ext}_{T}(F)=\sigma(F)$, where $\sigma(F)$ is the rank closure operator. Hence $\operatorname{ext}_{T}(F)$ is independent of the computation tree $T_{A}$. (In fact, this characterizes antimatroids among all greedoids - see Proposition 2.5 of [18].) Thus $\mathcal{C}_{F}$ is composed of the complements of the feasible sets of $\mathcal{F}_{\emptyset}$ for any computation tree $T_{A}$. Our first proposition simply translates the feasible set expansion of Proposition 3.1 into this setting.

Proposition 4.1 (Convex set expansion). Let $A$ be an antimatroid with free convex sets $\mathcal{C}_{F}$. Then

$$
\beta(A)=\sum_{K \in \mathcal{C}_{F}}(-1)^{|K|-1}|K| .
$$

Proof. If $T=T_{A}$ is any computation tree for $A$, then it follows from Theorem 2.5 of [18] that $\operatorname{ext}_{T}(F)=\emptyset$ precisely when $E-F$ is a free convex set. Then by Proposition 3.1, we have

$$
\begin{aligned}
\beta(A) & =(-1)^{r(A)} \sum_{F \in \mathcal{F}_{\emptyset}}(-1)^{|F|-1}(r(A)-|F|) \\
& =(-1)^{n} \sum_{K \in \mathcal{C}_{F}}(-1)^{n-|K|-1}|K| \\
& =\sum_{K \in \mathcal{C}_{F}}(-1)^{|K|-1}|K| .
\end{aligned}
$$


8 The EleCtronic Journal of COMBInatorics 4 (1997), \#R13

If $A$ is an antimatroid and $S \subseteq E$, then there is a unique smallest convex set which contains $S$ (see Section 8.7 of [5]), Define the convex closure operator $\tau(S)$ by

$$
\tau(S)=\bigcap_{C \in \mathcal{C}}\{C: S \subseteq C\}
$$

Then it is straightforward to verify $r(A-S)=|A-\tau(S)|$. This observation leads to the next expansion for $\beta(A)$.

Proposition $4.2(\tau(S)$ expansion). Let $A$ be an antimatroid. Then

$$
\beta(A)=\sum_{S \subseteq E}(-1)^{|S|-1}|\tau(S)| .
$$

Proof. From Proposition 2.1

$$
\begin{aligned}
\beta(A) & =(-1)^{r(A)} \sum_{S \subseteq E}(-1)^{|S|} r(S) \\
& =(-1)^{n} \sum_{S \subseteq E}(-1)^{|A-S|} r(A-S) \\
& =(-1)^{n} \sum_{S \subseteq E}(-1)^{|A-S|}|A-\tau(S)| \\
& =n \sum_{S \subseteq E}(-1)^{|S|}-\sum_{S \subseteq E}(-1)^{|S|}|\tau(S)| \\
& =\sum_{S \subseteq E}(-1)^{|S|-1}|\tau(S)| .
\end{aligned}
$$

The next result gives a different kind of expansion for the characteristic polynomial $p(A ; \lambda)$. In particular, we give a combinatorial interpretation to the coefficients of $p(A ; \lambda)$ when this polynomial is written in terms of the basis $\left\{(\lambda+1)^{k}\right\}_{k \geq 0}$.

Proposition 4.3. Let $A$ be an antimatroid with $r(A)=n$ and let $f_{k}$ be the number of intervals in $\mathcal{C}_{F}$ which are isomorphic to the Boolean algebra $B_{k}$. Then

$$
p(A ; \lambda)=(-1)^{n} \sum_{k=0}^{n}(-1)^{k} f_{k}(\lambda+1)^{k} .
$$

Proof. The semilattice $\mathcal{C}_{F}$ is meet-distributive. If $g_{k}$ denotes the number of elements of $\mathcal{C}_{F}$ which cover exactly $k$ elements of $\mathcal{C}_{F}$, then $g_{n-k}=w_{k}^{+}$, the (unsigned) $k^{t h}$ Whitney number. Thus, $g_{k}$ is the number of free convex sets of size $k$. By Proposition 8 of $[19]$ we get $p(A ; \lambda)=(-1)^{n} \sum_{k=0}^{n}(-1)^{k} g_{k} \lambda^{k}$. Then problem 19, page 156 of [26] gives the result.

Corollary 4.4. Let $f_{k}$ be the number of intervals in $\mathcal{C}_{F}$ which are isomorphic to the Boolean algebra $B_{k}$. Then

$$
\beta(A)=\sum_{k>0}(-2)^{k-1} k f_{k} .
$$

The next result gives an expansion for $\beta(A)$ for an antimatroid $A$ which is similar to the Möbius function formulation for a matroid. (See Section 7.3 of [28],) Let $\mu(C, D)$ denote the Möbius function on the lattice $(\mathcal{C}, \subseteq)$. 
Proposition 4.5 ( Möbius function). Let $A$ be an antimatroid and let $(\mathcal{C}, \subseteq)$ be the lattice of convex sets. Then

$$
\beta(A)=-\sum_{C \in \mathcal{C}} \mu(\emptyset, C)|C| .
$$

Proof. This follows from Theorem 1 of [19] and the definition of $\beta(A)$.

Recall that if $G=(E, \mathcal{F})$ is a greedoid and $S \subseteq E$, then the restriction of $G$ to $S$, written $G \mid S$, is a greedoid on the ground set $S$ whose feasible sets are just the feasible sets of $G$ which are contained in $S$. Equivalently, $G \mid S=G-(E-S)$. Note that when $A$ is an antimatroid, $A \mid S$ is an antimatroid precisely when $S$ is a feasible set.

We can prove the next result by applying Möbius inversion in $(\mathcal{C}, \subseteq)$ or by applying Proposition 11 of [19].

Proposition 4.6. Let $A=(E, \mathcal{F})$ be an antimatroid. Then

$$
\sum_{\emptyset \neq F \in \mathcal{F}} \beta(A \mid F)=n
$$

We end this section by translating Corollary 3.6 in the convex setting.

Proposition 4.7. Let $A$ be an antimatroid and let $\mathcal{M}$ be any perfect matching in $\left(\mathcal{C}_{F}, \subseteq\right)$. Let $M_{1} \subseteq \mathcal{C}_{F}$ denote the set of all feasible sets which are the minimal elements of the matching $\mathcal{M}$ and let $M_{2} \subseteq \mathcal{C}_{F}$ denote the set of all feasible sets which are the maximal elements of $\mathcal{M}$. Then

1. $\beta(A)=\sum_{C \in M_{1}}(-1)^{|C|}$,

2. $\beta(A)=\sum_{C \in M_{2}}(-1)^{|C|-1}$.

It is interesting to note that the expansions for $\beta(A)$ in terms of convex subsets generally have a simpler form than other expansions. In particular, the forms given for $\beta(A)$ in Propositions 4.1, 4.2, 4.5, and 4.7 seem especially compact.

\section{Simplicial SHELLiNG IN CHORDAL GRAPHS}

We now apply $\beta$ to the class of chordal graphs. Let $G$ be a chordal graph, i.e., a graph in which every cycle of length strictly greater than 3 has a chord. A vertex $v$ is called simplicial is its neighbors form a clique. Every chordal graph has at least two simplicial vertices [20] Then we get an antimatroid structure $A(G)$ on the vertex set $V$ by repeatedly eliminating simplicial vertices, i.e., $F \subseteq V$ is feasible if there is some ordering of the elements of $F$, say $\left\{v_{1}, v_{2}, \ldots, v_{k}\right\}$, so that for all $i(1 \leq i \leq k), v_{i}$ is simplicial in $G-\left\{v_{1}, \ldots, v_{i-1}\right\}$. This process of repeatedly removing simplicial verties is called simplicial shelling.

Let $b(G)$ be the number of blocks of the chordal graph $G$. (A block is a maximal subgraph which contains no cut-vertex.) The main theorem of this section is the following.

Theorem 5.1. Let $G$ be a connected chordal graph. Then $\beta(G)=1-b(G)$.

The proof of the theorem will follow several preliminary lemmas.

Lemma 5.1. $K \subseteq V$ is a free convex set if and only if the vertices of $K$ form a clique in $G$. 
10 The EleCtronic Journal of COMBInATORICS 4 (1997), \#R13

Proof. Suppose $K \subseteq V$ is a free convex set. Then $V-K$ is feasible (since $K$ is convex), and all subsets of vertices containing $V-K$ are also feasible (since $K$ is free). We write $K=\left\{v_{1}, v_{2}, \ldots, v_{r}\right\}$. Then, for all $i(1 \leq i \leq r)$, the set $(V-K) \cup\left\{v_{i}\right\}$ feasible means $v_{i}$ is simplicial in the induced subgraph on $K$, i.e., the vertices $\left\{v_{1}, \ldots, v_{i-1}, v_{i+1}, \ldots, v_{r}\right\}$ form a clique. Thus, the vertices of $K$ form a clique in $G$.

Now let $K$ be a clique in $G$. We must show that $K$ is convex. (It is clear that if $K$ is convex, then it must also be free.) Let $B_{d}(v)$ denote the closed ball of radius $d$ about $v$. By $(2.2)$ of $[20], B_{d}(v)$ is convex. Hence, $K=\bigcap_{v \in K} B_{1}(v)$ is also convex (since the intersection of convex sets is convex).

We now interpret greedoid deletion and contraction for chordal graphs. When $G$ is a chordal graph, we write $A(G)$ for the antimatroid corresponding to $G$ (as above). Thus, if $v$ is a simplicial vertex in $G$, we can perform the greedoid operations of deletion and contraction, yielding new antimatroids $A(G)-v$ and $A(G) / v$, respectively. The next result describes the convex sets in each of these antimatroids. We omit the straightforward proof.

Lemma 5.2. Let $v$ be a simplicial vertex in a chordal graph $G$ (with associated antimatroid $A(G)$ ) and let $C \subseteq V(G)$ with $v \notin C$. Then

1. $C$ is convex in $A(G) / v$ iff $C$ is convex in $A(G)$.

2. $C$ is convex in $A(G)-v$ iff $C \cup\{v\}$ is convex in $A(G)$.

This lemma allows us to interpret deletion and contraction of the simplicial vertex $v$ in terms of the chordal graph $G$. By Lemma 5.2(1), the antimatroid structure on $A(G) / v$ is isomorphic to the antimatroid structure on the chordal graph $G-v$, i.e., the graph $G$ with the vertex $v$ (and all incident edges) removed. Thus $A(G) / v \cong A(G-v)$. Deletion is more problematic for these antimatroids; in general, there is no chordal graph $H$ with $A(H)$ isomorphic to the deletion antimatroid $A(G)-v$. In spite of this difficulty, Lemma 5.2(2) still provides a graphical interpretation for $A(G)-v$.

To simplify notation, we will write $G / v$ instead of $A(G) / v$ and $G-v$ instead of $A(G)-v$. Since $r(G-v)=r(G)-1$ for any simplicial vertex $v$, we get the following:

Lemma 5.3. Let $v$ be a simplicial vertex in a chordal graph $G$. Then

$$
\beta(G)=\beta(G / v)-\beta(G-v) .
$$

The next result follows immediately from Lemmas 5.1 and 5.2(2).

Lemma 5.4. Let $v$ be a simplicial vertex in a chordal graph $G$. Then $K$ is a free convex set in $G-v$ iff $K \cup\{v\}$ forms a clique in $G$.

The next result follows from the definition of a simplicial vertex.

Lemma 5.5. Let $v$ be a simplicial vertex in a chordal graph $G$ which is a block. Then $G / v$ is also a block.

Lemma 5.6. Let $G$ be a chordal graph (with at least one edge) which is a block. Then $\beta(G)=0$.

Proof. We proceed by induction on $n=|V|$. If $n<2$, then $G$ is not a block. Thus we begin the induction with $n=2$. But then $G$ must be an edge, and it is easy to see $\beta(G)=0$. 
Let $v$ be a simplicial vertex. Then by Lemma 5.3, we have $\beta(G)=\beta(G / v)-$ $\beta(G-v)$. By Lemma $5.5, G / v$ is a block, so $\beta(G / v)=0$ by induction. It remains to show $\beta(G-v)=0$. Let $N(v) \subseteq V$ be the vertices adjacent to $v$ in $G$ and write $m=|N(v)|$. Since $v$ is simplicial, $N(v)$ forms a clique. By Propostion 4.1 and Lemma 5.4 we have

$$
\begin{aligned}
\beta(G-v) & =\sum_{K \subseteq N(v)}(-1)^{|K|-1}|K| \\
& =\sum_{k=0}^{m}(-1)^{k-1} k\left(\begin{array}{c}
m \\
k
\end{array}\right) \\
& =0
\end{aligned}
$$

since $m>1$. Thus $\beta(G)=0$.

Our last lemma describes how $\beta$ behaves under two graph constructions.

Lemma 5.7. Let $G$ be a chordal graph.

1. If $G_{1}$ and $G_{2}$ are connected components of $G$, then $\beta(G)=\beta\left(G_{1}\right)+\beta\left(G_{2}\right)$.

2. If $G_{1}$ and $G_{2}$ are subgraphs of $G$ with exactly one vertex $v$ in common, then $\beta(G)=\beta\left(G_{1}\right)+\beta\left(G_{2}\right)-1$.

Proof. (1) Let $\mathcal{K}$ denote the family of all (vertex sets of) cliques of $G$ and $\mathcal{K}_{i}$ the cliques of $G_{i}$ (for $i=1,2$ ). By Lemma 5.1 and Proposition 4.1.

$$
\begin{aligned}
\beta(G) & =\sum_{K \in \mathcal{K}}(-1)^{|K|-1}|K| \\
& =\sum_{K \in \mathcal{K}_{1}}(-1)^{|K|-1}|K|+\sum_{K \in \mathcal{K}_{2}}(-1)^{|K|-1}|K| \\
& =\beta\left(G_{1}\right)+\beta\left(G_{2}\right) .
\end{aligned}
$$

(2) This is exactly the same as part 1 , except the clique $\{v\}$ is counted twice in the sum $\beta\left(G_{1}\right)+\beta\left(G_{2}\right)$. This clique contribute 1 to each block; thus $\beta(G)=$ $\beta\left(G_{1}\right)+\beta\left(G_{2}\right)-1$.

We are now ready to prove Theorem 5.1.

Proof. Suppose $G$ is composed of $k$ blocks. We proceed by induction on $k$. If $k=1$, then the result follows by Lemma 5.6. Now assume $k \geq 2$ and let $B$ be a block which contains exactly one cut-vertex of $G$. (Such a block always exists - see Theorem 2.15 of [10]]. Let $v$ be this cut-vertex of $G$ and perform the operation of vertex splitting at $v$ to obtain a new graph $H$ with exactly two connected components $B$ and $C$ (as in Figure 2).

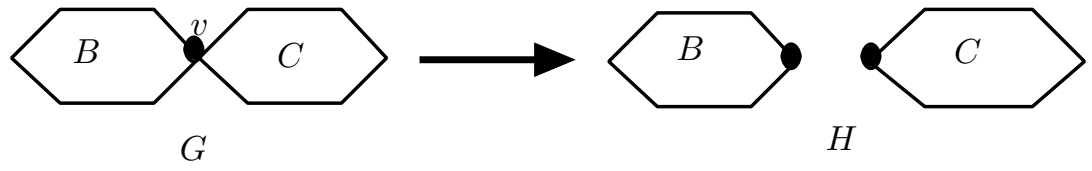

FiguRe 2.

Now $\beta(G)=\beta(B)+\beta(C)-1$ by Lemma 5.7(2). By Lemma 5.6, $\beta(B)=0$. By induction, since $C$ is composed of $k-1$ blocks, $\beta(C)=1-(k-1)$. Combining these equations gives us $\beta(G)=1-k$, as required. 


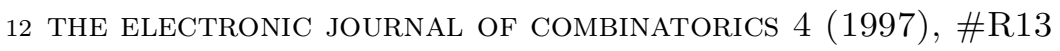

Corollary 5.8. Let $G$ be a tree with $n$ edges. Then $\beta(G)=1-n$.

Proof. Each edge of a tree is a block, so the result follows from the theorem.

There are several possibilities for future research in this area. Computing $\beta(G)$ for other classes of greedoids, e.g., rooted graphs, rooted digraphs, trees, posets (both the single and double shelling antimatroids) and convex point sets in Euclidean space should prove worthwhile. We mention one result[1]] in this context: If $C$ is a finite set of points in the plane, then define an antimatroid $A(C)$ as follows [23]: $K \subseteq C$ is convex iff $K=H \cap C$ for some (ordinary) convex subset of the plane $H$. Then $\beta(C)$ equals the number of points in $C$ which are interior to the convex hull of $C$. 


\section{REFERENCES}

[1] C. Ahrens, G. Gordon and E. McMahon, Finite subsets of the plane and the $\beta$ invariant, in preparation.

[2] M. Aigner, Whitney numbers, in Combinatorial Geometries (N. White, ed.), Encyclopedia of Mathematics and Its Applications, Vol. 29, pp. 139-160, Cambridge Univ. Press, London, 1987.

[3] A. Björner, Homology amd shellability of matroids and geometric lattices, in Matroid Applications (N. White, ed.), Encyclopedia of Mathematics and Its Applications, Vol. 40, pp. 226-283, Cambridge Univ. Press, London, 1992.

[4] A. Björner and G. Ziegler, Broken circuit complexes: Factorizations and generalizations, J. Comb. Theory (B) 51 (1991), 96-126.

[5] A. Björner and G. Ziegler, Introduction to Greedoids, in Matroid Applications (N. White, ed.), Encyclopedia of Mathematics and Its Applications, Vol. 40, pp. 284-357, Cambridge Univ. Press, London, 1992.

[6] T. Brylawski, A combinatorial model for series-parallel networks, Trans. Amer. Math. Soc., 154 (1971), 1-22.

[7] T. Brylawski, The broken-circuit complex, Trans. Amer. Math. Soc., 234 (1977), 417-433.

[8] T. Brylawski and J. Oxley, The broken-circuit complex: Its structure and factorizations, Eur. J. Comb. 2 (1981), 107-121.

[9] T. Brylawski and J. Oxley, The Tutte Polynomial and its Applications, in Matroid Applications (N. White, ed.), Encyclopedia of Mathematics and Its Applications, Vol. 40, pp. 123-225, Cambridge Univ. Press, London, 1992.

[10] G. Chartrand and L. Lesniak, Graphs \& Digraphs, Second Edition, Wadsworth \& Brooks/Cole, Monterey, CA, 1986.

[11] S. Chaudhary and G. Gordon, Tutte polynomials for trees, J. Graph Theory, 15 (1991), 317-331.

[12] H. Crapo, A higher invariant for matroids, J. Comb. Theory 2 (1967), 406-417.

[13] P. Edelman and R. Jamison, The theory of convex geometries, Geom. Ded. 19 (1985), 247270.

[14] G. Gordon, A Tutte polynomial for partially ordered sets, J. Comb. Theory (B) 59 (1993), 132-155.

[15] G. Gordon, Series-parallel posets and the Tutte polynomial, Discrete Math. 158 (1996), 63-75.

[16] G. Gordon, E. McDonnell, D. Orloff and N. Yung, On the Tutte polynomial of a tree, Cong. Numer., 108 (1995), 141-151.

[17] G. Gordon and E. McMahon, A greedoid polynomial which distinguishes rooted arborescences, Proc. Amer. Math. Soc., 107 (1989), 287-298.

[18] G. Gordon and E. McMahon, Interval partitions and activities for the greedoid Tutte polynomial, Adv. in Appl. Math. 18 (1997), 33-49.

[19] G. Gordon and E. McMahon, A greedoid characteristic polynomial, Contemp. Math. 197 (1996), 343-351.

[20] R. E. Jamison-Waldner, A perspective on abstract convexity: Classifying alignments by varieties, in Convexity and Related Combinatorial Geometry, (D. C. Kay and M. Breen, eds.), Marcel Dekker, Inc., New York (1982).

[21] E. McMahon, On the greedoid polynomial for rooted graphs and rooted digraphs, J. Graph Theory, 17 (1993), 433-442.

[22] B. Monjardet, A use for frequently rediscovering a concept, Order 1 (1985), 415-417.

[23] B. Korte, L. Lovsz and R. Schrader, Greedoids, Springer-Verlag, Berlin, 1991.

[24] J. Oxley, On Crapo's beta invariant for matroids, Stud. in Appl. Math. 66 (1982), 267-277.

[25] R. Stanley, Acyclic orientations of graphs, Discrete Math. 5 (1973), 171-178.

[26] R. Stanley, Enumerative Combinatorics, Vol. 1, Wadsworth \& Brooks/Cole, Monterey, CA, 1986.

[27] W. T. Tutte, A contribution to the theory of chromatic polynomials, Can. J. Math. 6, 80-91.

[28] T. Zaslavsky, The Möbius function and the characteristic polynomial, in Combinatorial Geometries (N. White, ed.), Encyclopedia of Mathematics and Its Applications, Vol. 29, pp. 114-138, Cambridge Univ. Press, London, 1987.

Department of Mathematics, Lafayette College, Easton, PA 18042

E-mail address: gordong@lafayette.edu 